\title{
MerTK receptor cleavage promotes plaque necrosis and defective resolution in atherosclerosis
}

\author{
Bishuang Cai, ${ }^{1}$ Edward B. Thorp, ${ }^{2}$ Amanda C. Doran, ${ }^{1}$ Brian E. Sansbury, ${ }^{3}$ Mat J.A.P. Daemen, ${ }^{4}$ Bernhard Dorweiler, ${ }^{5}$ \\ Matthew Spite, ${ }^{3}$ Gabrielle Fredman, ${ }^{6}$ and Ira Tabas ${ }^{1,7}$ \\ 'Department of Medicine, Columbia University, New York, New York, USA. ²Department of Pathology, Feinberg Cardiovascular Research Institute, Northwestern University, Chicago, Illinois, USA. \\ ${ }^{3}$ Center for Experimental Therapeutics and Reperfusion Injury, Brigham and Women's Hospital and Harvard Medical School, Boston, Massachusetts, USA. ${ }^{4}$ Academic Medical Center, Department of Pathology, \\ Cardiovascular Research, Amsterdam, Netherlands. ${ }^{5}$ Department of Cardiothoracic and Vascular Surgery, Universitätsmedizin Mainz, Mainz, Germany. ${ }^{6}$ Department of Molecular and Cellular Physiology, \\ Center for Cardiovascular Sciences, Albany Medical College, Albany, New York, USA. `Department of Pathology and Cell Biology and Department of Physiology, Columbia University, New York, New York, USA.
}

\begin{abstract}
Atherothrombotic vascular disease is often triggered by a distinct type of atherosclerotic lesion that displays features of impaired inflammation resolution, notably a necrotic core and thinning of a protective fibrous cap that overlies the core. A key cause of plaque necrosis is defective clearance of apoptotic cells, or efferocytosis, by lesional macrophages, but the mechanisms underlying defective efferocytosis and its possible links to impaired resolution in atherosclerosis are incompletely understood. Here, we provide evidence that proteolytic cleavage of the macrophage efferocytosis receptor c-Mer tyrosine kinase (MerTK) reduces efferocytosis and promotes plaque necrosis and defective resolution. In human carotid plaques, MerTK cleavage correlated with plaque necrosis and the presence of ischemic symptoms. Moreover, in fat-fed LDL receptor-deficient ( $\left(\mathrm{dll}^{-/-}\right)$mice whose myeloid cells expressed a cleavage-resistant variant of MerTK, atherosclerotic lesions exhibited higher macrophage MerTK, lower levels of the cleavage product soluble Mer, improved efferocytosis, smaller necrotic cores, thicker fibrous caps, and increased ratio of proresolving versus proinflammatory lipid mediators. These findings provide a plausible molecular-cellular mechanism that contributes to defective efferocytosis, plaque necrosis, and impaired resolution during the progression of atherosclerosis.
\end{abstract}

\section{Introduction}

Atherothrombotic vascular disease is the leading cause of death in the industrialized world. While the majority of atherosclerotic lesions are clinically silent, acute cardiovascular events can be triggered by lesions with plaque necrosis, thin collagenous "caps" covering necrotic cores, and increased inflammation (1). We and others have highlighted the role of 2 processes: defective efferocytosis, which leads to postapoptotic necrosis; and an impaired inflammation resolution response, which contributes to persistent plaque inflammation, fibrous cap thinning, and thrombosis (2-5). Interestingly, efferocytosis has been linked to resolution of inflammation (6-8), which suggested to us an integrated pathophysiologic hub in the formation of clinically dangerous plaques.

Here we focus on a macrophage efferocytosis receptor called c-Mer tyrosine kinase (MerTK), which mediates efferocytosis in atherosclerotic lesions $(9,10)$, has been linked to resolution $(8)$, and can be cleaved by ADAM17 $(8,11,12)$. Moreover, macrophages near the necrotic core of human atheromas were shown to have lower MerTK and higher ADAM17 than peripheral lesional macrophages (13). In this context, we hypothesized that MerTK cleavage promotes defective efferocytosis and impaired resolution in atherosclerosis.

Conflict of interest: The authors have declared that no conflict of interest exists. Submitted: September 2, 2016; Accepted: November 22, 2016.

Reference information: J Clin Invest. 2017;127(2):564-568

https://doi.org/10.1172/JCI90520.

\section{Results and Discussion}

We analyzed cross sections of carotid artery endarterectomy specimens from 14 patients for soluble Mer (sol-Mer), a marker of MerTK cleavage (11, 12), and plaque necrosis and found that lesional sol-Mer was positively correlated with necrosis (Figure 1A and Supplemental Figure 1A; supplemental material available online with this article; doi:10.1172/JCI90520DS1). Sol-Mer was not detected in nonatherosclerotic human arteries. We next compared carotid plaques from additional subjects who either had suffered a transient ischemic attack or stroke (symptomatic) or were asymptomatic. Plaques of symptomatic patients had higher levels of sol-Mer compared with those of asymptomatic patients (Figure 1B), and plasma sol-Mer was also increased in symptomatic patients (Supplemental Figure 1B).

MerTK cleavage causes defective MerTK-mediated efferocytosis $(8,11,12)$. A previous study showed that recombinant sol-Mer can inhibit efferocytosis in vitro, presumably through competition for molecules that bridge apoptotic cells (ACs) to MerTK, like GAS6 (11). However, we found that recombinant sol-Mer inhibited efferocytosis only at doses that were more than 1,000-fold those in plasma (Supplemental Figure 1C). Thus, unless sol-Mer concentrations in lesions reach extremely high levels, sol-Mer is most likely a marker rather than a cause of defective MerTK efferocytosis. In this context, we found that cell-surface MerTK in macrophage-rich areas in plaques from symptomatic patients was less than that in asymptomatic patients (Figure 1C) despite sim- 

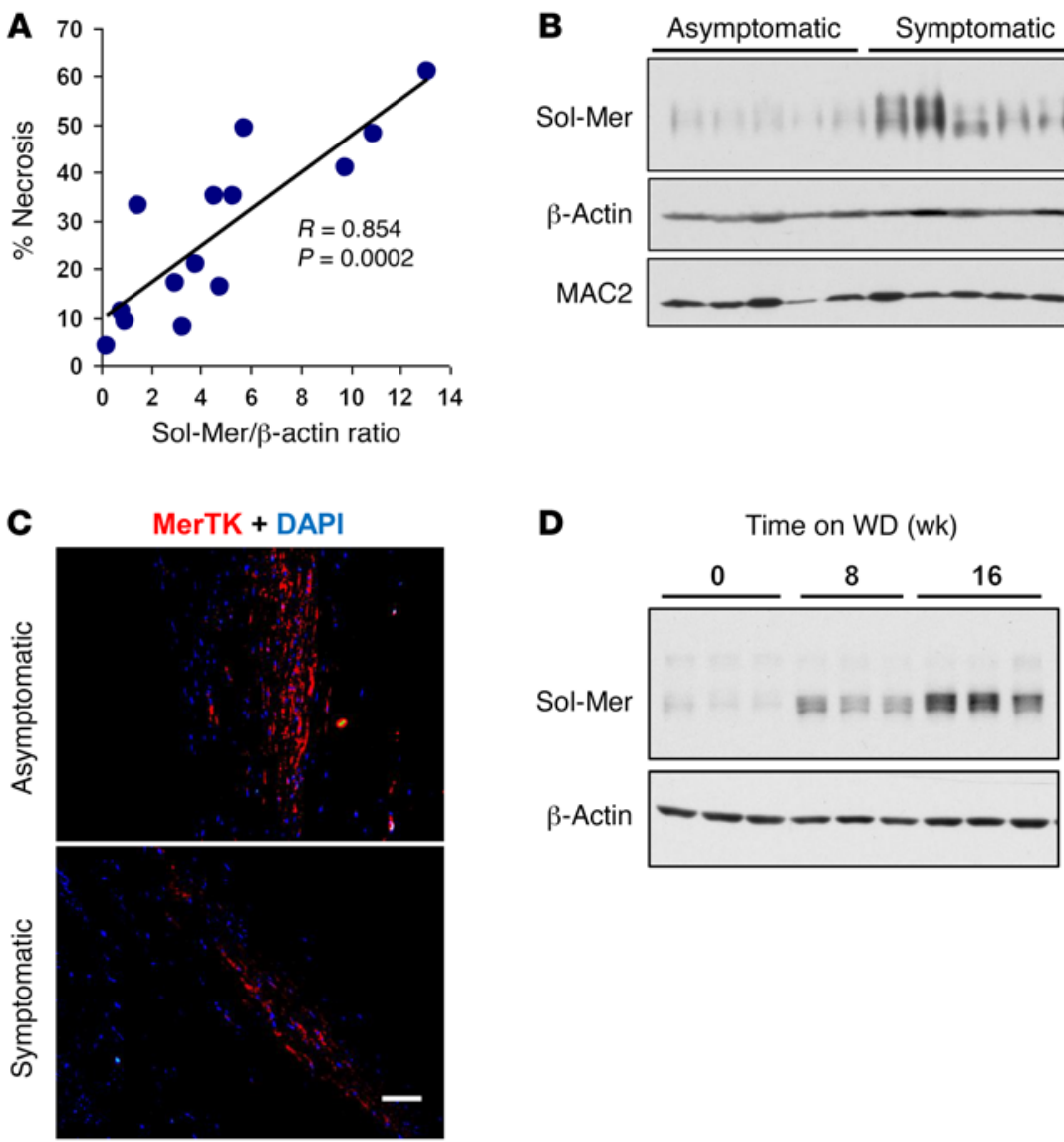

Figure 1. Sol-Mer is increased in advanced human and murine atherosclerotic lesions. (A) Carotid endarterectomy (CEA) specimens were analyzed for sol-Mer by immunoblot, and necrotic area was measured from $\mathrm{H} \& \mathrm{E}$-stained images. The correlation coefficient $(R)$ and $P$ value were based on Pearson's product-moment correlation analysis. (B) CEA specimens from asymptomatic and symptomatic patients were assessed by immunoblot for sol-Mer ( $n=5$ per group). $\beta$-Actin and MAC2 were used as loading controls for total protein and macrophages, respectively. (C) Paraffin-embedded CEA sections were stained with anti-MerTK antibody, imaged by confocal microscopy (scale bar: $30 \mu \mathrm{m}$ ), and quantified by MerTK mean fluorescence intensity (MFI) relative to the asymptomatic cohort ( $n=6-8$ for each group). ${ }^{*} P<0.05$, by 2 -tailed Student's $t$ test. (D) Age-matched $L d l r^{-1-}$ mice were fed the Western-type diet (WD) for 0,8 , or 16 weeks and sacrificed at 24 weeks of age. The aortic arch, Brachiocephalic Artery (BCA), and descending aorta up to the renal bifurcation were removed en bloc and immunoblotted for sol-Mer ( $n=3$ per group).

To study advanced atherosclerosis, WT or Mertk $^{C R}$ bone marrow was transplanted into $\mathrm{Ldlr}^{-/}$ mice, and atherosclerotic lesions and plasma were analyzed after 16 weeks of WD feeding. These 2 groups had similar body weight, blood glucose, plasma lipids, and circulating leukocytes (Supplemental Figures 3 and 4). We confirmed that aortic sol-Mer was reduced and that MerTK was higher in the CR cohort (Figure 2, A and B). Most importantly, Mertk ${ }^{C R}$ lesions had substantially less plaque necrosis but no change in total lesion area or lesional cell composition (Figure 2C and Supplemental Figure 5), indicating a true decrease in percentage necrosis. Further, Mertk ${ }^{C R}$ lesions had a markedly lower ratio of free to macrophage-associated $\mathrm{TUNEL}^{+}$ (apoptotic) cells (Figure 2D), which reflects improved efferocytosis.

ilar macrophage content (Supplemental Figure 1D). Finally, aortic sol-Mer in LDL receptor-deficient ( $\left.\mathrm{ddlr}^{--}\right)$mice increased as a function of time on Western-type diet (WD) (Figure 1D), which correlates with plaque progression (14).

To test the hypothesis that MerTK cleavage promotes plaque necrosis, we used a mouse model in which endogenous Mertk was replaced with a genetically engineered mutant gene encoding cleavage-resistant MerTK (Mertk $\left.{ }^{C R}\right)$ (8). Cleavage-resistant MerTK is functional under basal conditions but, unlike WT MerTK, maintains full activity under cleavage-promoting conditions, e.g., in the presence of inflammatory stimuli (8). We show here that the atherogenic lipoprotein oxidized LDL (oxLDL) increased sol-Mer and decreased cell-surface MerTK in control (WT) macrophages but not in macrophages pretreated with the ADAM17 inhibitor TAPI-0 (Supplemental Figure 2A) or in macrophages isolated from Mertk $k^{C R}$ mice (Supplemental Figure 2, B and C). Importantly, oxLDL suppressed efferocytosis in WT but not Mertk ${ }^{C R}$ macrophages (Supplemental Figure 2D).
Mertk ${ }^{C R}$ lesions also had thicker fibrous cap thickness and increased collagen gene expression (Figure 3, A and B), which are associated with plaque stability and inflammation resolution $(1,15)$. These lesions also had more Tregs (Figure 3C), which can promote resolution $(16,17)$, are decreased in human and mouse advanced atheroma (18), and have been causatively linked to plaque progression in mice (19).

Defective inflammation resolution can be caused by an imbalance between specialized proresolving mediators (SPMs) and proinflammatory lipid mediators in both human and mouse lesions $(20,21)$. Using liquid chromatography-tandem mass spectrometry to assay a wide range of SPMs and proinflammatory lipid mediators in aortic extracts, we found that the global content of SPMs, including resolvin D2, D5, and E3, was significantly increased in Mertk $^{C R}$ extracts (Supplemental Table 1 and Figure 3D).

The inflammation resolution program has a number of positive-feedback processes. First, SPMs can increase production of proteins that dampen inflammation and promote repair $(22,23)$. 
A

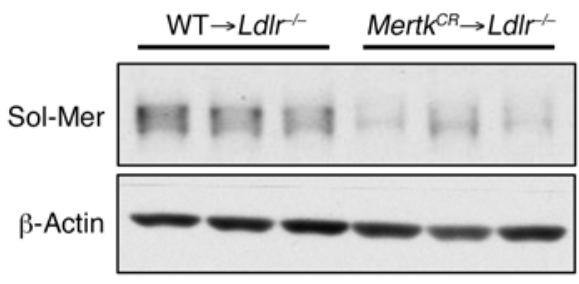

B
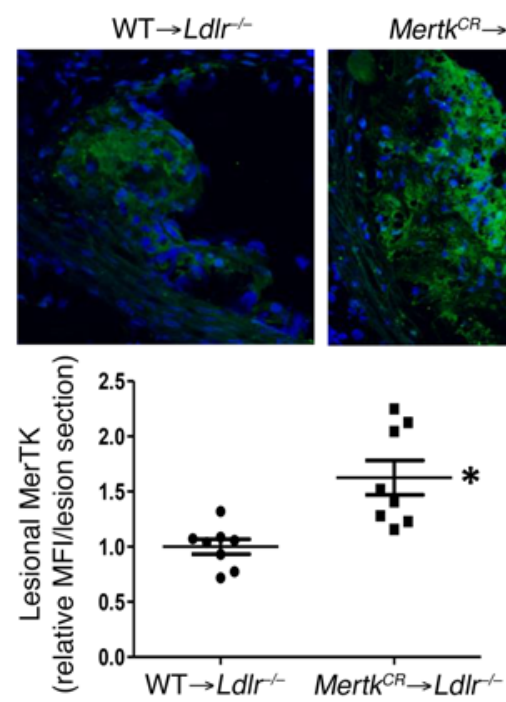

C
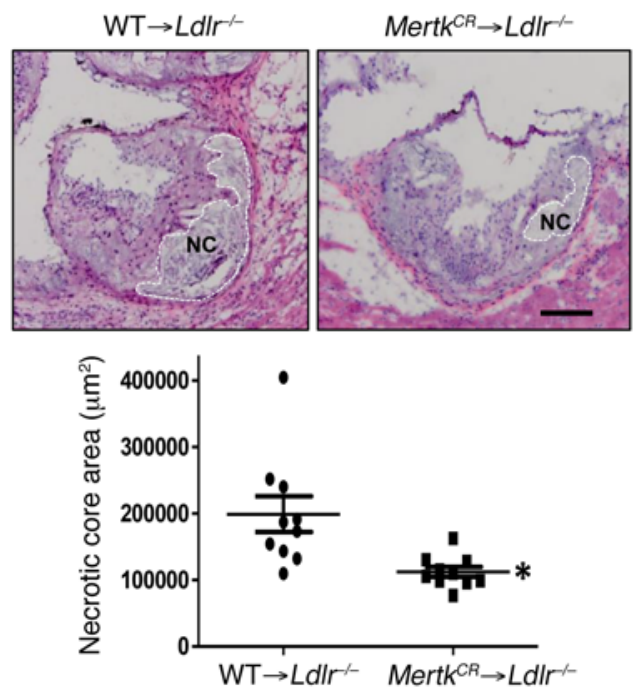

D

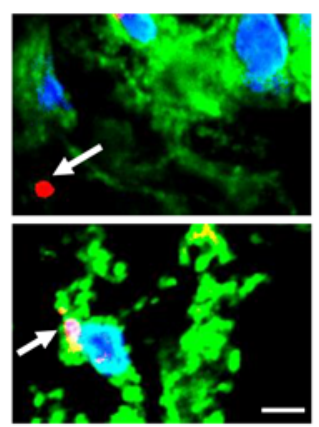

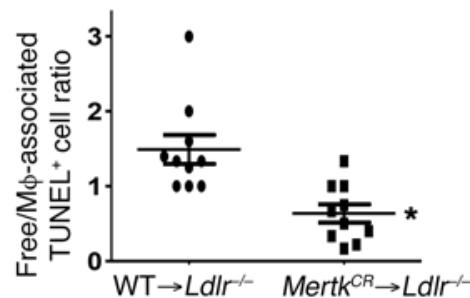

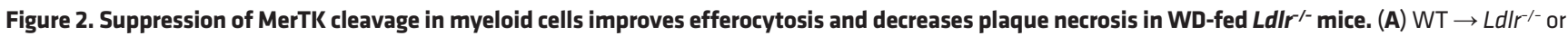
Mert $^{C R} \rightarrow L d l r^{\prime-}$ bone marrow-transplanted mice were fed WD for 16 weeks. Aortas were harvested and analyzed for sol-Mer as described in Figure $1 \mathrm{D}$ ( $n=3$ per group). ${ }^{*} P<0.05$. (B) Confocal microscopy of MerTK in aortic root cross sections (scale bar: $20 \mu \mathrm{m}$ ). Images were quantified as MerTK MFI relative to the WT $\rightarrow$ Ldlr $r^{-1-}$ cohort ( $n=8$ per group). ${ }^{*} P<0.01$. (C) Representative H\&E images of aortic root sections, with necrotic core (NC) regions indicated by broken lines (scale bar: $40 \mu \mathrm{m})$, and quantification of necrotic core area ( $n=10$ per group, 2 independent experiments). ${ }^{*} P<0.01$. (D) Representative images of aortic root sections in which apoptotic cells were labeled by TUNEL (red), macrophages by anti-F4/80 (green), and nuclei by Hoechst (blue) (scale bar: $10 \mu \mathrm{m})$. The white arrows depict apoptotic cells that were either free (top image) or associated with macrophages (bottom image). The graph shows quantification of the ratio of free to macrophage-associated apoptotic cells ( $n=10$ for each group, 2 independent experiments). ${ }^{*} P<0.05$. A 2-tailed Student's $t$ test was used for all panels.

Two such proteins, TGF- $\beta 1$ and IL-10, were significantly increased in Mertk $^{C R}$ plasma (Supplemental Figure 6A). Second, SPMs can upregulate their own receptors (24), and we found that the expression of the SPM receptor ALX/FPR2 was markedly induced in the lesions of Mertk ${ }^{C R}$ mice (Supplemental Figure 6B). Third, engagement of MerTK by apoptotic cells and other ligands in cultured macrophages and in sterile inflammation in vivo enhances the synthesis of 5-LOX-derived SPMs at the expense of inflammatory leukotrienes $(8,25)$. We therefore analyzed the ratio of 5 -LOX-derived SPMs to leukotrienes and found that this ratio was markedly increased in Mertk $^{C R}$ extracts (Figure 3E). Moreover, this ratio was negatively correlated with necrotic core area (Figure $3 \mathrm{~F}$ ). Fourth, using bone marrow-derived macrophages, we found that resolvin D1 (RvD1) blocks LPS-induced MerTK cleavage (Supplemental Figure 7, A and B). Importantly, when WD-fed $\mathrm{Ldlr}^{-/}$mice were treated with RvD1, which suppresses plaque necrosis and improves lesional efferocytosis (20), lesional MerTK expression was increased (Supplemental Figure 7C). Thus, MerTK cleavage during plaque progression suppresses the integrated processes of efferocytosis and inflammation resolution and disrupts several components of positive-feedback signaling. These findings likely explain the marked improvement in plaque morphology in the atherosclerotic lesions of $\mathrm{Mertk}^{C R} \rightarrow L d l r^{-/}$mice.

Understanding processes involved in the formation of the unique types of atherosclerotic plaques that cause acute cardiovascular events is a critical goal in cardiovascular research. Progression to plaque necrosis is a key process because of the highly inflammatory and plaque-destabilizing nature of the necrotic core (26). Data from human and mouse studies suggest that plaque necrosis results from 2 complementary processes, postapoptotic necrosis secondary to defective efferocytosis as examined here $(2,3)$ and a primary cell necrosis process called necroptosis $(27,28)$. While the current study is focused on an efferocytosis defect in phagocytes themselves, a recent study showed that some apoptotic cells in human and mouse lesions inappropriately retain CD47, which blocks their engulfment by normal phagocytes (29). Interestingly, oxLDL, which was shown here to promote MerTK cleavage and defective efferocytosis, can induce necroptosis in macrophages, and these cells are also poorly 

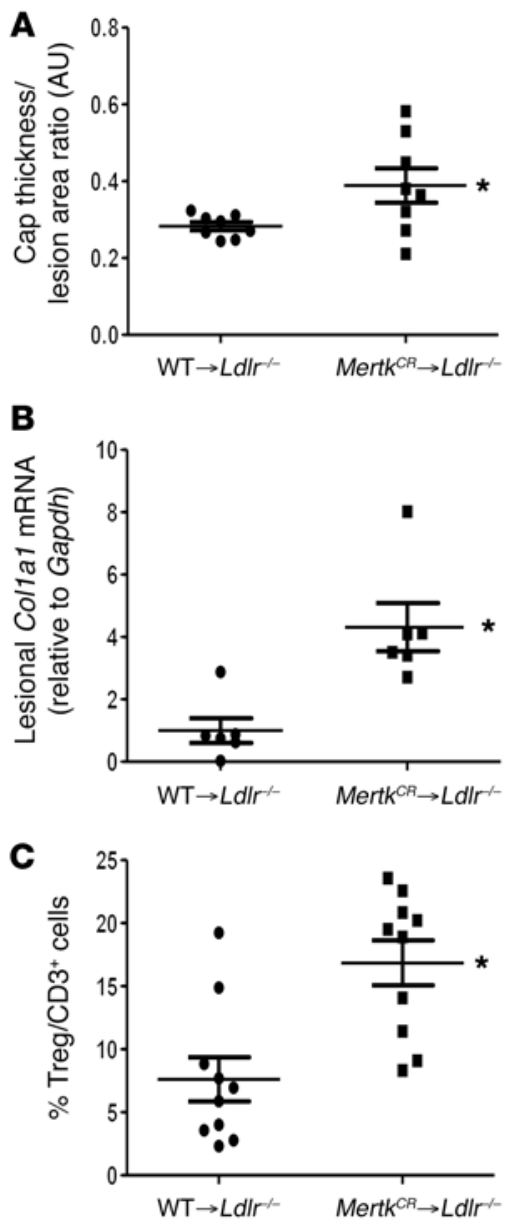

D

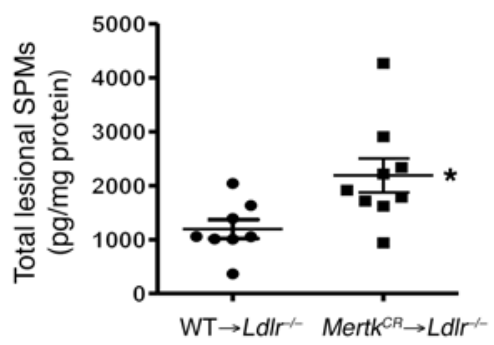

E

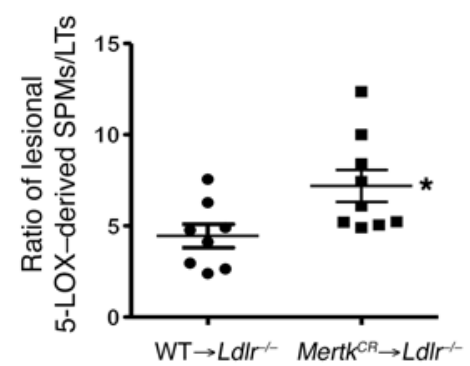

$\mathbf{F}$

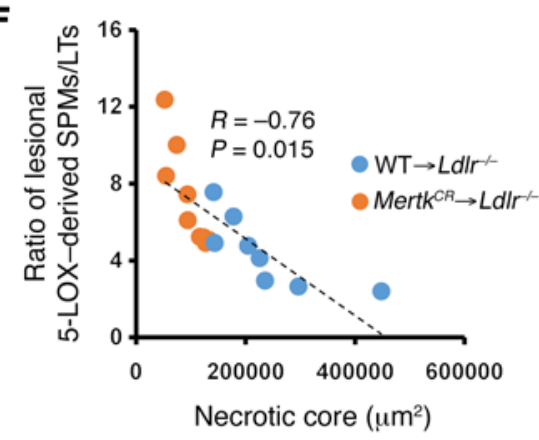

Figure 3. Suppression of MerTK cleavage improves features of resolution in plaques and increases aortic content of specialized proresolving mediators. (A) Aortic root sections from WT $\rightarrow$ LdIr $^{-/}$and Mertk ${ }^{C R} \rightarrow$ $\mathrm{LdII}^{\text {/- }}$ bone marrow-transplanted mice were stained with Picrosirius red. Quantified data are presented as the ratio of fibrous cap thickness to lesion area, expressed as arbitrary units (AU) ( $n=8$ per group). ${ }^{*} P<0.05$. (B) A subset of aortic root sections was chosen randomly for quantification of Col1a1 mRNA by reverse transcriptase quantitative $P C R$, with normalization to Gapdh mRNA ( $n=6$ for each group). ${ }^{*} P<0.05$. (C) FoxP3 ${ }^{+}$Tregs and total $\mathrm{CD3}^{+} \mathrm{T}$ cells were quantified in aortic root sections by immunofluorescence microscopy and expressed as percentage Tregs/CD3 ${ }^{+}$cells ( $n=10$ for each group). The average absolute numbers of these cells, most of which were in the adventitia, were $8 \pm 2.49$ and $19 \pm 3.74$ per section for FoxP3 ${ }^{+}$Tregs ( $\left.{ }^{*} P<0.05\right)$ and $106 \pm 16.13$ and $107 \pm 14.33$ per section for $\mathrm{CD}^{+}$cells (NS) for the WT and Mertk ${ }^{\mathrm{CR}}$ cohorts, respectively. ( $\mathbf{D}$ and $\mathbf{E}$ ) Quantification of specialized proresolving mediators (SPMs) and ratio of aortic 5-LOX-derived SPMs/leukotrienes (LTs) in aorta of WT $\rightarrow$ LdIr $^{\prime-}(n=8)$ versus Mertk ${ }^{C R} \rightarrow$ LdIr $^{\prime-}(n=9)$ bone marrow-transplanted mice. ${ }^{*} P<0.05$. (F) Correlation of the ratio of 5-LOX-derived SPMs/leukotrienes with necrotic core area $(n=17)$. $P$ represents the 2 -tailed probability value of a Pearson correlation coefficient. $A$ 2-tailed Student's $t$ test was used for A-E. internalized by normal phagocytes (28). Defects in multiple steps of efferocytosis, together with activation of necroptotic pathways in advanced plaques, would create a "perfect storm" for the development of plaque necrosis.

Our data support the concept that MerTK plays an integral part in a program of feedback signaling that amplifies resolution. This concept aligns with previous studies showing that (a) engagement of MerTK by apoptotic cells or other activators can activate both antiinflammatory and proresolving pathways (6-8, 30-32); and (b) proresolving mediators can promote MerTK expression and efferocytosis $(7,33,34)$. Thus, when MerTK is rendered inactive by proteolytic cleavage, not only is there a defect in efferocytosis, but positive-feedback signaling in resolution is impaired, leading to loss of resolving mediators and further defects in efferocytosis. In the setting of advanced atherosclerosis, this profound deficit promotes key features of clinically dangerous plaques, including necrosis, imbalance of proresolving versus proinflammatory lipid mediators, fibrous cap thinning, and deficiency of Tregs. The finding that cleavage of a single molecule in the setting of a chronic inflammatory condition could have such robust pathophysiologic effects not only suggests its potential as a therapeutic target but also raises the question of the possible role of MerTK cleavage in other chronic inflammatory conditions previously linked to MerTK and sol-Mer, e.g., systemic lupus erythematosus and Sjögren's syndrome (35-38).

\section{Methods}

A complete description of methods is provided in the Supplemental Methods.

Statistics. Data are displayed as mean \pm SEM. Because all data in this study fit a normal distribution, a 2-tailed Student's $t$ test was used to determine statistical significance. $P$ values less than 0.05 were considered significant. For the data in Figure 1A, correlation coefficient $(R)$ and $P$ value were calculated using Pearson product-moment correlation analysis.

Study approval. For human studies, carotid endarterectomy samples were obtained from M.J.A.P. Daemen, Academic Medical Center, Amsterdam, the Netherlands, and Bernhard Dorweiler, University Medical Center, Johannes-Gutenberg University, Mainz, Germany. Use of all material conformed with the declaration of Helsinki and was approved by the appropriate university ethics review board. Complete details are available in the Supplemental Methods. All animals were cared for according to NIH and IACUC guidelines in a barrier facility at Columbia University Medical Center, New York, New York, USA.

\section{Author contributions}

BC, EBT, GF, and IT conceived and designed the research. MJAPD and $\mathrm{BD}$ donated patient samples and were instrumental in the interpretation of the human data. BC, EBT, BES, MS, and GF conducted the experiments. BC, EBT, ACD, BES, GF, MS, and IT analyzed the data. BC, ACD, and IT wrote the paper. 


\section{Acknowledgments}

We thank Ying Wang for assistance with the bone marrow transplantation experiments. This work was supported by an American Heart Association postdoctoral fellowship (to BC), NIH grant T32 HL007854-21 (to ACD), NIH R00 grant HL119587 (to GF), American Federation for Aging Research grant A16034 (to GF), and NIH grants HL106173 and GM095467 (to MS) and HL132412, HL075662, and HL127464 (to IT).

Address correspondence to: Ira Tabas, Columbia University, 630 W. 168th Street, New York, New York 10032, USA. Phone: 212.305.9430; E-mail: iat1@columbia.edu.
1. Virmani R, Kolodgie FD, Burke AP, Farb A, Schwartz SM. Lessons from sudden coronary death: a comprehensive morphological classification scheme for atherosclerotic lesions. Arterioscler Thromb Vasc Biol. 2000;20(5):1262-1275.

2. Schrijvers DM, De Meyer GR, Kockx MM, Herman AG, Martinet W. Phagocytosis of apoptotic cells by macrophages is impaired in atherosclerosis. Arterioscler Thromb Vasc Biol. 2005;25(6):1256-1261.

3. Tabas I. Consequences and therapeutic implications of macrophage apoptosis in atherosclerosis: the importance of lesion stage and phagocytic efficiency. Arterioscler Thromb Vasc Biol. 2005;25(11):2255-2264.

4. Merched AJ, Ko K, Gotlinger KH, Serhan CN, Chan L. Atherosclerosis: evidence for impairment of resolution of vascular inflammation governed by specific lipid mediators. FASEB J. 2008;22(10):3595-3606.

5. Tabas I. Macrophage death and defective inflammation resolution in atherosclerosis. Nat Rev Immunol. 2010;10(1):36-46.

6. Schwab JM, Chiang N, Arita M, Serhan CN. Resolvin E1 and protectin D1 activate inflammation-resolution programmes. Nature. 2007;447(7146):869-874.

7. Mitchell S, et al. Lipoxins, aspirin-triggered epi-lipoxins, lipoxin stable analogues, and the resolution of inflammation: stimulation of macrophage phagocytosis of apoptotic neutrophils in vivo. J Am Soc Nephrol. 2002;13(10):2497-2507.

8. Cai B, et al. MerTK cleavage limits proresolving mediator biosynthesis and exacerbates tissue inflammation. Proc Natl Acad Sci US A. 2016;113(23):6526-6531.

9. Thorp E, Cui D, Schrijvers DM, Kuriakose G, Tabas I. Mertk receptor mutation reduces efferocytosis efficiency and promotes apoptotic cell accumulation and plaque necrosis in atherosclerotic lesions of apoe-/- mice. Arterioscler Thromb Vasc Biol. 2008;28(8):1421-1428.

10. Ait-Oufella H, et al. Defective mer receptor tyrosine kinase signaling in bone marrow cells promotes apoptotic cell accumulation and accelerates atherosclerosis. Arterioscler Thromb Vasc Biol. 2008;28(8):1429-1431.

11. Sather S, et al. A soluble form of the Mer receptor tyrosine kinase inhibits macrophage clearance of apoptotic cells and platelet aggregation. Blood. 2007;109(3):1026-1033.

12. Thorp E, Vaisar T, Subramanian M, Mautner L, Blobel C, Tabas I. Shedding of the Mer tyrosine kinase receptor is mediated by ADAM17 protein through a pathway involving reactive oxygen species, protein kinase $\mathrm{C} \delta$, and p38 mitogen-ac- tivated protein kinase (MAPK). J Biol Chem. 2011;286(38):33335-33344.

13. Garbin U, et al. Expansion of necrotic core and shedding of Mertk receptor in human carotid plaques: a role for oxidized polyunsaturated fatty acids? Cardiovasc Res. 2013;97(1):125-133.

14. Ishibashi S, Goldstein JL, Brown MS, Herz J, Burns DK. Massive xanthomatosis and atherosclerosis in cholesterol-fed low density lipoprotein receptor-negative mice. J Clin Invest. 1994;93(5):1885-1893.

15. Fredman G, et al. Targeted nanoparticles containing the proresolving peptide Ac2-26 protect against advanced atherosclerosis in hypercholesterolemic mice. Sci Transl Med. 2015;7(275):275ra20.

16. Krishnamoorthy N, et al. Cutting edge: maresin-1 engages regulatory $\mathrm{T}$ cells to limit type 2 innate lymphoid cell activation and promote resolution of lung inflammation. JImmunol. 2015;194(3):863-867.

17. Gagliani N, et al. Th17 cells transdifferentiate into regulatory $\mathrm{T}$ cells during resolution of inflammation. Nature. 2015;523(7559):221-225.

18. Dietel B, Cicha I, Voskens CJ, Verhoeven E, Achenbach S, Garlichs CD. Decreased numbers of regulatory $\mathrm{T}$ cells are associated with human atherosclerotic lesion vulnerability and inversely correlate with infiltrated mature dendritic cells. Atherosclerosis. 2013;230(1):92-99.

19. Foks AC, et al. Differential effects of regulatory $T$ cells on the initiation and regression of atherosclerosis. Atherosclerosis. 2011;218(1):53-60.

20. Fredman G, et al. An imbalance between specialized pro-resolving lipid mediators and pro-inflammatory leukotrienes promotes instability of atherosclerotic plaques. Nat Commun. 2016;7:12859.

21. Viola JR, et al. Resolving Lipid Mediators Maresin 1 and Resolvin D2 Prevent Atheroprogression in Mice. Circ Res. 2016;119(9):1030-1038.

22. Serhan CN, Chiang N, Van Dyke TE. Resolving inflammation: dual anti-inflammatory and pro-resolution lipid mediators. Nat Rev Immunol. 2008;8(5):349-361.

23. Serhan CN, Krishnamoorthy S, Recchiuti A, Chiang N. Novel anti-inflammatory - pro-resolving mediators and their receptors. Curr Top Med Chem. 2011;11(6):629-647.

24. Simiele F, et al. Transcriptional regulation of the human FPR2/ALX gene: evidence of a heritable genetic variant that impairs promoter activity. FASEB J. 2012;26(3):1323-1333.

25. Fredman G, et al. Resolvin D1 limits 5-lipoxygenase nuclear localization and leukotriene B4 synthesis by inhibiting a calcium-activated kinase pathway. Proc Natl Acad Sci U S A.
2014;111(40):14530-14535.

26. Bentzon JF, Otsuka F, Virmani R, Falk E. Mechanisms of plaque formation and rupture. Circ Res. 2014;114(12):1852-1866.

27. Lin J, et al. A role of RIP3-mediated macrophage necrosis in atherosclerosis development. Cell Rep. 2013;3(1):200-210.

28. Karunakaran D, et al. Targeting macrophage necroptosis for therapeutic and diagnostic interventions in atherosclerosis. Sci Adv. 2016;2(7):e1600224.

29. Kojima Y, et al. CD47-blocking antibodies restore phagocytosis and prevent atherosclerosis. Nature. 2016;536(7614):86-90.

30. Tibrewal N, et al. Autophosphorylation docking site Tyr-867 in Mer receptor tyrosine kinase allows for dissociation of multiple signaling pathways for phagocytosis of apoptotic cells and down-modulation of lipopolysaccharide-inducible NF- $\kappa \mathrm{B}$ transcriptional activation. J Biol Chem. 2008;283(6):3618-3627.

31. Rothlin CV, Ghosh S, Zuniga EI, Oldstone MB, Lemke G. TAM receptors are pleiotropic inhibitors of the innate immune response. Cell. 2007;131(6):1124-1136.

32. Freire-de-Lima CG, Xiao YQ, Gardai SJ, Bratton DL, Schiemann WP, Henson PM. Apoptotic cells, through transforming growth factor- $\beta$, coordinately induce anti-inflammatory and suppress pro-inflammatory eicosanoid and NO synthesis in murine macrophages. J Biol Chem. 2006;281(50):38376-38384.

33. Li Y, Dalli J, Chiang N, Baron RM, Quintana C, Serhan CN. Plasticity of leukocytic exudates in resolving acute inflammation is regulated by MicroRNA and proresolving mediators. Immunity. 2013;39(5):885-898.

34. Serhan CN, Savill J. Resolution of inflammation: the beginning programs the end. Nat Immunol. 2005;6(12):1191-1197.

35. Scott RS, et al. Phagocytosis and clearance of apoptotic cells is mediated by MER. Nature. 2001;411(6834):207-211.

36. Wu J, et al. Increased plasma levels of the soluble Mer tyrosine kinase receptor in systemic lupus erythematosus relate to disease activity and nephritis. Arthritis Res Ther. 2011;13(2):R62.

37. Zizzo G, Guerrieri J, Dittman LM, Merrill JT, Cohen PL. Circulating levels of soluble MER in lupus reflect M2c activation of monocytes/macrophages, autoantibody specificities and disease activity. Arthritis Res Ther. 2013;15(6):R212.

38. Qin B, et al. The association of Tyro3/Axl/Mer signaling with inflammatory response, disease activity in patients with primary Sjögren's syndrome. Joint Bone Spine. 2015;82(4):258-263. 